\title{
MoBIE: A free and open-source platform for integration and cloud-based sharing of multi-modal correlative big image data
}

Christian Tischer ${ }^{1}$, Constantin Pape ${ }^{1}$, Kimberly Meechan ${ }^{1}$, Valentyna Zinchenko ${ }^{1}$, Martin Schorb ${ }^{1}$, Hernando Vergara $^{2}$, Detlev Arendt ${ }^{1}$, Anna Kreshuk ${ }^{1}$ and Yannick Schwab ${ }^{1}$

${ }^{1}$ EMBL Heidelberg, United States, ${ }^{2}$ Sainsbury Wellcome Centre, United States

Recently, we presented the registration of a whole-body cellular expression atlas to a high-resolution electron microscopy (EM) dataset, automatic segmentation of all cell somata and nuclei, and clustering of the cells according to gene expression or morphological parameters [1]. This dataset is a valuable resource containing rich biological information. However, the size and complexity of this holistic resource (currently 231 image sources adding up to $2.02 \mathrm{~TB}$ lossless compressed image data, including EM, light microscopy, segmentation images and segment feature tables) poses a challenge to its effective interrogation for scientific discovery. To integrate this dataset we developed MoBIE: a free and open-source platform for multi-modal big image data exploration and sharing. MoBIE (https://github.com/mobie/mobie) consists of an object store backend for cloud-based image data sharing, GitHub based storage of tabular data, and an easy to install Fiji [3] plugin for integrated browsing of the whole dataset (Fig. 1). The image data is stored in a compressed, chunked and object store compatible data format (https://github.com/saalfeldlab/n5) where only the chunks needed for the current rendering are downloaded into the Fiji viewer. In addition, the image data is stored as a resolution pyramid enabling exploration at different scales. As a consequence, even TB-sized image data can be smoothly explored at arbitrary orientations and zoom levels on a standard computer. In addition, we provide interactive viewing modalities for image segmentations, including segment feature tables, segment feature scatterplots and segment volume rendering (Fig. 1). All viewers are linked, sharing the same image segments selection and coloring model. It soon became apparent that, in addition to [1], also other datasets including various modalities such as EM tomography [5] can be efficiently shared using this platform. In fact, within few months MoBIE transformed the way we integrate and share multi-modal big image data at our institute. We are therefore excited to present this platform as we hope that it will facilitate multi-modal image data exploration and sharing also at other institutions. 

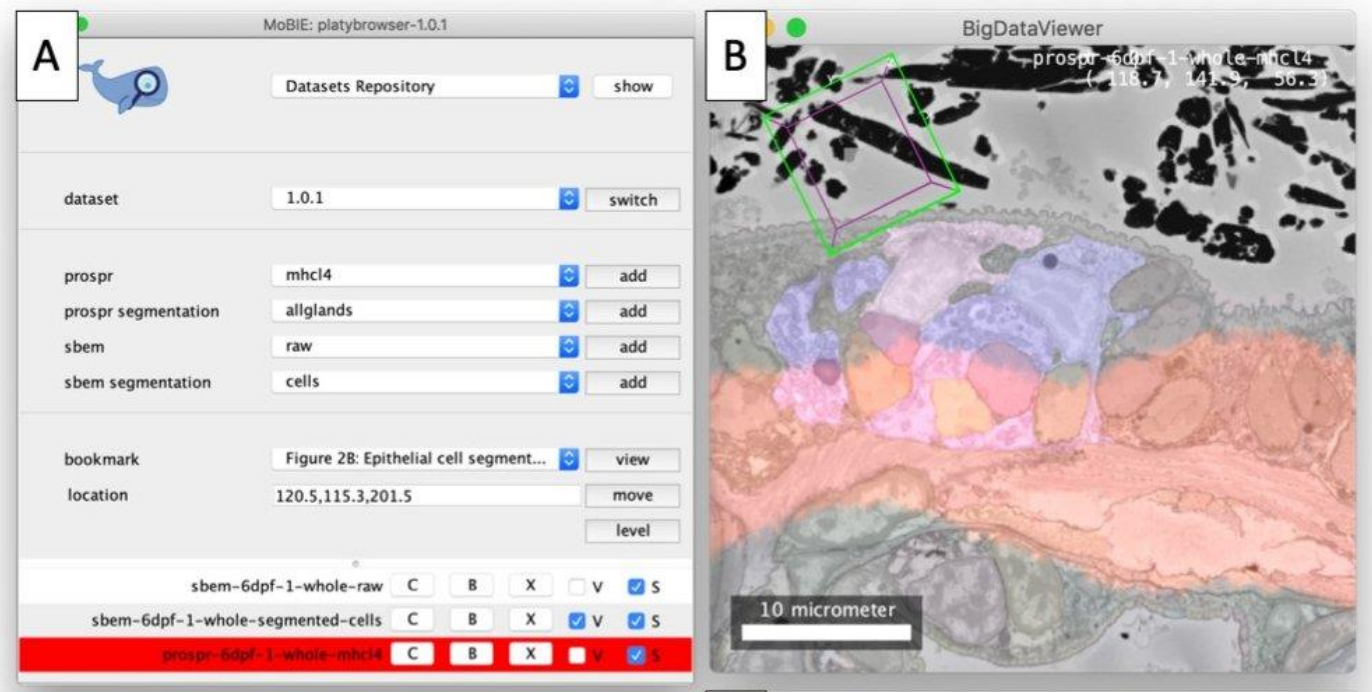

\begin{tabular}{|c|c|c|}
\hline & sbe & \\
\hline id & anchor_ $x$ & bb \\
\hline .0 & $151.0679 \ldots 183.0456 \ldots 35.64595 \ldots 147.3$ & 18 \\
\hline 2738.0 & $151.7996 \ldots 180.0293 \ldots 37.72238 \ldots 147.76$ & 17 \\
\hline 2739.0 & $155.6380 \ldots 180.5414 \ldots 35.39347 \ldots 147.32$ & 17 \\
\hline 2740.0 & $160.5117 \ldots 181.5662 \ldots 33.45219 \ldots 157.74$ & 18 \\
\hline 2741.0 & $164.1546 \ldots 178.8996 \ldots 37.79130 \ldots 161.12$ & 17 \\
\hline 2742.0 & $167.3957 \ldots 182.8466 \ldots 36.57437 \ldots 164.44$ & 17 \\
\hline 2743.0 & $166.2307 \ldots 188.2630 \ldots 36.52947 \ldots 158.26$ & 18 \\
\hline 2744.0 & $169.1884 \ldots 181.5498 \ldots 34.82836 \ldots 167.18$ & 17 \\
\hline 2745.0 & $188.7005 \ldots 184.9254 \ldots 36.76219 \ldots 183.92$ & 17 \\
\hline 2746.0 & $196.8526 \ldots 186.5935 \ldots 36.40120 \ldots 190.18$ & 18 \\
\hline 2747.0 & $198.5047 \ldots 185.0020 \ldots 37.93982 \ldots 194.28$ & 18 \\
\hline 2748.0 & $220.8893 \ldots 176.2387 \ldots 35.92156 \ldots 217.5$ & 17 \\
\hline 2749.0 & $234.7543 \ldots 180.0244 \ldots 33.58729 \ldots 232.54$ & 17 \\
\hline 2750.0 & $234.7253 \ldots 182.8664 \ldots 34.20980 \ldots 233.12$ & 17 \\
\hline 2751.0 & $133.3815 \ldots 193.4894 \ldots 34.36808 \ldots 132.32$ & 19 \\
\hline 2752.0 & $133.8256 \ldots 190.7781 \ldots 32.64916 \ldots 133.12$ & 18 \\
\hline 2753.0 & $160.3645 \ldots 192.0235 \ldots 35.59821 \ldots 157.9$ & 18 \\
\hline 2754.0 & $157.0338 \ldots 191.6978 \ldots 37.74045 \ldots 153.6$ & 18 \\
\hline 2755.0 & $167.3207 \ldots 192.1917 \ldots 33.51878 \ldots 165.58$ & 19 \\
\hline 2756.0 & $172.6988 \ldots 190.1409 \ldots 39.44073 \ldots 168.18$ & 18 \\
\hline 2757.0 & $171.2687 \ldots 192.5450 \ldots 34.99931 \ldots 169.9200 \ldots$ & 19 \\
\hline 2758.0 & $180.8422 \ldots 189.0182 \ldots 35.63361 \ldots 178.0$ & 18 \\
\hline & & \\
\hline
\end{tabular}

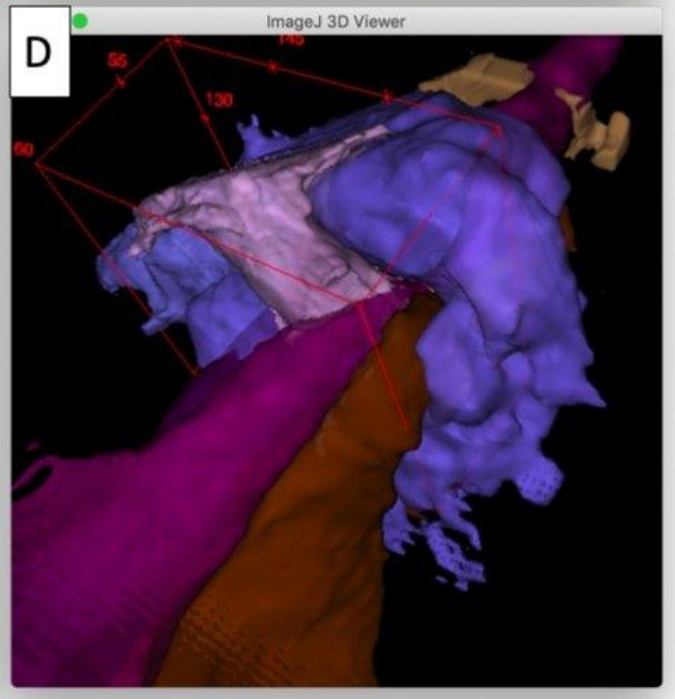

Figure 1. Screenshots of the MoBIE Fiji plugin. A) User interface for browsing the image sources. B) BigDataViewer [3] window for interactive 2-D slice viewing of multi-modal, multi-scale image data. C) Interactive image segments feature table. D) ImageJ 3-D Viewer [4] for interactive image segments volume rendering.

\section{References}

[1] Vergara et al., "Whole-body integration of gene expression and single-cell morphology", bioRxiv, https://doi.org/10.1101/2020.02.26.961037, 2020

[2] Schindelin et al., "Fiji: an open-source platform for biological-image analysis", 2012, Nat. Methods 9, 676682.

[3] Pietzsch et al., "BigDataViewer: visualization and processing for large image data sets", 2015, Nat. Methods 12, 481-483.

[4] Schmid et al., "A high-level 3D visualization API for Java and ImageJ", 2010, BMC Bioinformatics, 11(1): 1

[5] Cortese et al., "Integrative Imaging Reveals SARS-CoV-2-Induced Reshaping of Subcellular Morphologies", Cell Host \& Microbe, 2020, 28, 853-866.e5 